\title{
RoboTouch - An artificial Skin for Human-Robot Interaction
}

\author{
M. Fritzsche, N. Elkmann \\ Fraunhofer Institute for Factory Operation and Automation, \\ Sandtorstrasse 22, 39106 Magdeburg, Germany, \\ $<$ firstname>.<lastname>@iff.fraunhofer.de
}

\begin{abstract}
Beside the well-established robot applications in the range of industrial tasks, where the workspaces of humans and robots are strictly separated, new application areas for service and assistance robots are moving more and more into the focus of interest. Within these applications the robots are no longer banned behind strong steel fences but share a common workspace with the human being. The main advantage of such systems is to combine the flexibility and intelligence of the human being with the robots ability to fulfil monotonous tasks with high precision.
\end{abstract}

The interaction between humans and robots within a common workspace (e.g. in industrial, but also in household or laboratory environments) allows - and also often requires - physical contact between humans and robots. This human-robot interaction can bring many advantages, but it also poses a high risk potential for the human. Therefore the main task in developing such applications is to minimize the safety risk for the human.

An important contribution to this task has been made by the Fraunhofer IFF through the development of a pressure sensitive artificial skin, which will be further developed and certified as a collision detection sensor for safety applications. A patent has been applied for the sensor setup.

The sensor system is intended to be applied to robotic arms where standard collision detection sensors cannot be used. Covering the whole robot surface, the sensor system is able to capture forces affecting the sensor surface. Due to a matrix assembly of multiple sensor cells a pressure distribution may be determined with the sensor data. Through the use of intelligent postprocessing algorithms, impacts and collisions may be detected and an emergency stop signal is generated afterwards.

The measuring principle of the artificial skin sensor is based upon the pressure dependent resistance of conductive elastomeric foam. Via textile electrodes and textile ribbon cables a matrix setup is built out of single foam elements. Covered by a cladding material the sensor elements are protected against external influences. Due to this construction, consisting solely of textile materials, the sensor system is a highly durable.

As a result of the desired construction, existing problems such as reproducible coupling between electrodes and conductive foam and the protection of sensor elements against external influences could be solved.

Even though the main intention in developing the artificial skin sensor was to use it as a safety sensor for robotic applications, our experience indicates that the sensor has a wide range of possible applications including tactile input units, monitoring and survey units, measuring systems for pressure distributions and many other smart textile applications.

The first application of the sensor system is for the whole robotic system within the LiSA project, supported by the Federal Ministery of Education and Research (www.lisa-roboter.de).

Our experiences, the sensor technology itself and the ongoing development will be described in more detail within this paper. 


\section{Introduction}

In addition to well established robotic applications for industrial tasks, which strictly separate humans and robots' workspaces, new applications for service and assistance robots are increasingly attracting interest. In such applications, robots are no longer banned behind protective barriers but share common workspaces with humans. The main advantage of such systems is their combination of humans' flexibility and intelligence with robots' capability to perform monotonous tasks with high precision.

Human-robot interaction in shared workspaces, e.g. in industrial, household and lab environments, allows and also often requires physical contact between humans and robots. While such human-robot interaction can bring many advantages, it also poses a high risk potential for humans. Therefore, the main challenge when developing such applications is to minimize risks for humans.

The Fraunhofer Institute for Factory Operation and Automation IFF has made an important contribution by developing a pressure-sensitive artificial skin that is undergoing further development and will be certified as a collision detection sensor for safety applications. A patent has been filed for the sensor system.

The LiSA project offered the first significant opportunity to test the artificial skin sensor, which endows the LiSA robot with a sense of touch. This approach opens new strategies for collision avoidance and detection and also allows innovative methods of control employing tactile interaction.

\section{The artificial skin sensor}

The Fraunhofer IFF researches sensor principles for locally resolved detection of forces. The goal is to produce cost effective solutions with high measurement performance, thus also making such systems attractive for mass applications.

A novel conductive woven textile provides the basis for a matrix structure. Its crossover points are formed by a special conductive elastomer with pressure-dependent resistance. A compartmentalized jacket material fixes the matrix configuration and gives the individual sensor cells a defined pretensioning (Fig.1). The matrix rows and columns are electrically connected via textile ribbon cables.

[4] textile ribbon cable

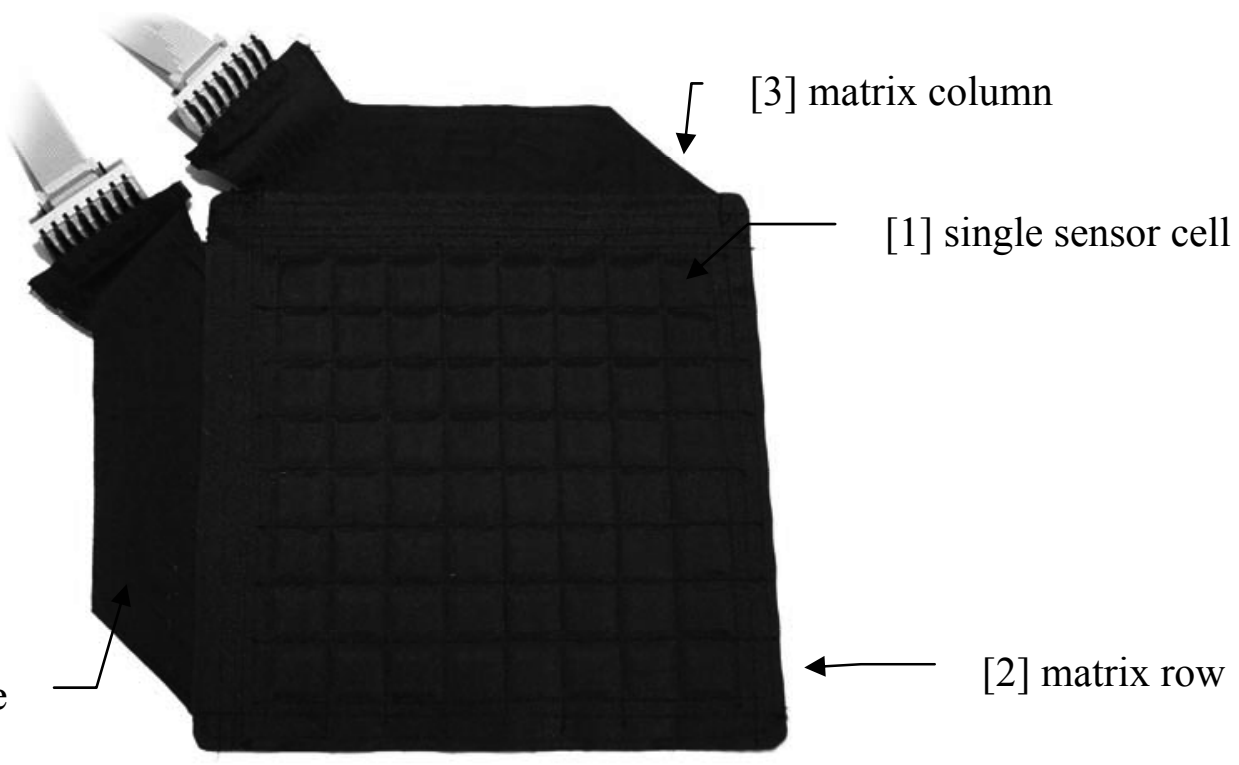

Fig. 1- Prototype $8 \times 8$ matrix sensor 
A microcontroller-based matrix controller addresses and monitors individual sensor cells' conductivity. If active forces compromise individual sensor cells, the affected cells' conductivity and thus the evaluable current flow changes. Consequently current I is a measure of the active force. The individual sensor cells' pretensioning enables measuring a bias current in an unloaded state too. This allows complete condition monitoring of the entire sensor system.

In conjunction with the matrix structure, the matrix controller ensures that cross currents between neighboring sensor cells are minimized. Thus, neighboring sensor cells' signals are represented completely decoupled (Fig. 2).

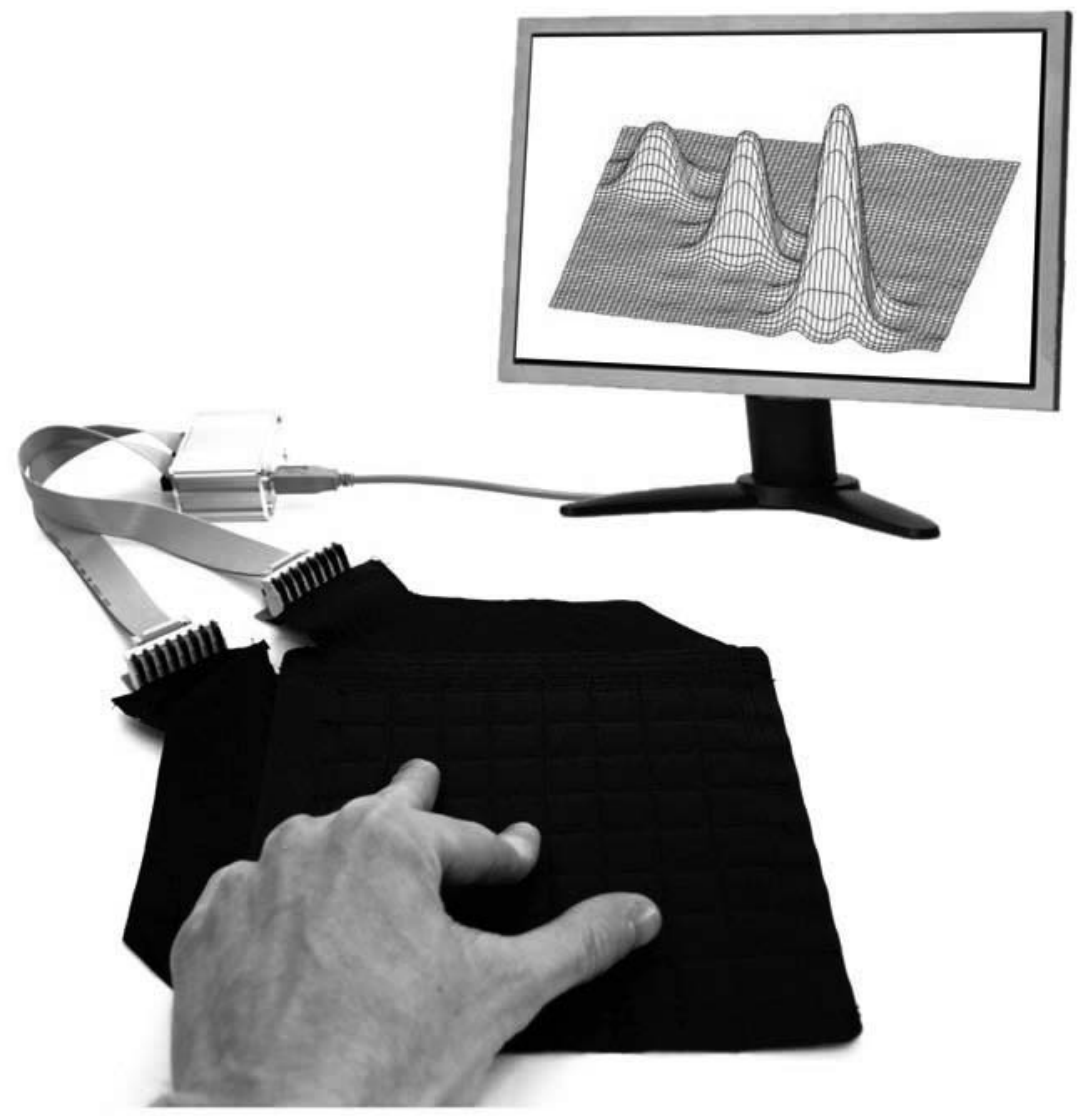

Fig. 2 - Visualization of 3-fingers touching the sensor, signal level represents the applied pressure

A USB and a CAN interface transmit the measured data to a superordinate control or PC. What is more, the CAN interface can connect several sensor units to a macro unit.

An additional feature, the matrix controller provides a switching output for safety applications. Independent of the data interfaces, the matrix controller can be integrated in a machine's emergency stop circuit and utilized for collision detection. The sensor system is being prepared for certification as a safety sensor compliant with the current standards.

The actual prototype matrix controller makes it possible to connect sensor fields with up to $32 \times 32$ cells. Larger sensor fields can be monitored by coupling several matrix controllers.

The Sensor systems spatial resolution depends on the cell size and the gaps between the single sensor cells. Limited by the design of the jacket material's chambers, the minimum cell size is approximately $5 \times 5$ $\mathrm{mm}$. This value also limits the achievable spatial resolution of the sensor system. To ensure condition monitoring is reliable, a maximum cell size of $75 \times 75 \mathrm{~mm}$ may not be exceeded.

2-3 $\mathrm{mm}$ thick and completely built up of textile, the sensor system represents a flexible, durable, and conformable solution that can be easily adapted to different geometries. Complex geometries can already be produced during manufacturing. 
The jacket material largely determines the sensor mat's mechanical properties. Apart from particularly rugged or watertight designs, air permeable and breathable sensor types are possible.

A patent has been filed for the sensor system and its related design variants. The Sensor system is still under development. Different design variants are going to be tested concerning to durability, limits and application areas. A first use case has been found within the LiSA project.

\section{The LiSA Project}

Partially funded by the German Federal Ministry of Education and Research (BMBF), the LiSA project is aimed at integrating the latest research findings in a service robot that meets the high level of safety demanded in real world human-robot collaboration.

The primary objective of the project has been the development and construction of an assistance robot suitable for daily use in a semi-structured life science lab environment. Designed as a logistics system, the robot should be able to autonomously execute transportation tasks without any risk to the humans working in the robot's workspace.

This scenario has great value for the robotic applications in the life sciences, a field that often entails monotonous or hazardous work or requires highly sterile conditions.

For more detailed information on the objectives of the project, the robot itself and the life science scenario, the authors refer readers to [1-3] or to the LiSA website: www.lisa-roboter.de.

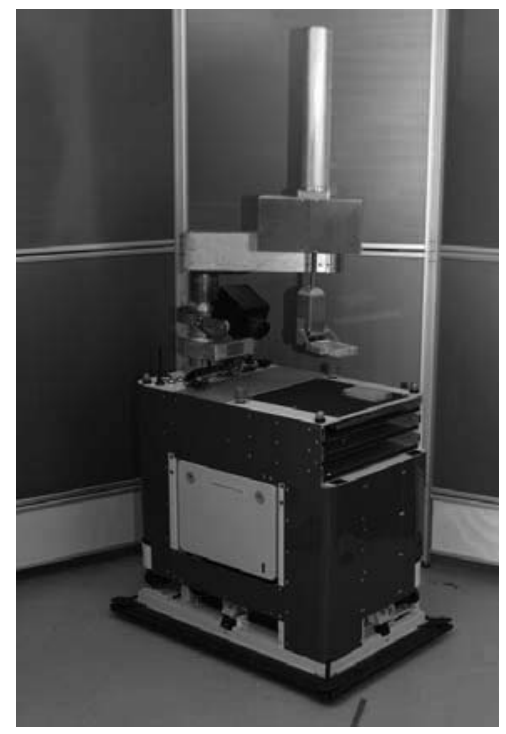

Fig. 3 - The LiSA robot

\section{LiSA and Its Artificial Skin}

As part of the LiSA project, the artificial skin is being implemented in the LiSA robot. Although not yet certified as a safety sensor, it primarily serves to protect the robotic arm. Moreover, its special features also qualify it as a haptic interface.

\section{A Safety Collision Sensor Based on the Artificial Skin Sensor}

The risk analysis performed for the LiSA robot identified the robot arm (see Fig.3) as the greatest source of hazards. Its geometry and the workspace generate a variety of trapping and collision points. Design engineering measures and path planning strategies can reduce some of the risks. Nonetheless, a residual risk always remains. This problem is nothing new. The KR-3 SI robot [6] is the first commercially 
available robot system approved for human-robot interaction. When the robot's approach is followed by contact, it safely stops. Another groundbreaking system is DLR's lightweight arm [7]. Complex hardware captures the forces and moments in the individual joints. Intelligent evaluation algorithms allow it to react to collisions and contact. An artificial skin implementable both for interaction and collision detection is described in [8, 9]. Another sensors system usable as an artificial robot skin is presented in [10]. Both systems utilize principles of resistive measurement.

A similar approach is being pursued in the LiSA project with the artificial skin developed at the Fraunhofer IFF. The sensor system completely encases the robotic arm to detect collisions between the robotic arm and its environment.

To this end, a damping layer is initially applied to the robotic arm, which absorbs the kinetic energy in the case of a collision and compensates for the required overtravel caused by the time lag between collision detection and the drives' stop.

Then, individual sensor mats adapted to the geometry are mounted and each is furnished with a matrix controller. A CAN bus connects the individual matrix controllers to a virtual macro sensor that covers the entire arm.

In the robot's automatic mode, the sensor system initially operates in "collision detection mode". If a collision is detected, a switching signal is generated, which initiates one of four possible user-defined reactions:

a) The robot switches the power in the drives off so that the holding brakes engage and the robotic arm is blocked. This strategy represents a classic emergency stop scenario. However, this approach only appears conditionally suited for human-robot interaction since the arm could irreversibly seize.

b) The robot switches the power in the drives off but does not engage the brakes. Depending on how the gears have locked, the user can move the robotic arm manually and loosen possible seizing. However, this strategy is only suitable for a SCARA robot. A buckling arm robot would collapse.

c) The robot stops the current movement and shifts the sensor system's mode from "collision detection mode" to "haptic device mode". The forces acting on the sensor system are interpreted as control impulses. This enables the user to also move the robotic arm manually and loosen possible seizing. This strategy can be employed independent of the robot's design.

d) The robot stops the current movement and attempts to dissipate the active forces through appropriate evasive movements. However, this strategy holds the risk of additional dangers from the evasive movements.

Regardless of the strategy selected, the robot only resumes its original movement after acknowledgement from the user.

\section{The Artificial Skin as a Haptic Device}

The use of the artificial skin as a haptic interface allows using contact to directly guide the robot. This is not only expedient to terminate collisions but also to teach or intervene in motion sequences.

To do so, the sensor system is switched to the "haptic device mode". If the user then only exerts pressure on one robot segment, a resultant force vector is calculated on the basis of the active forces and the geometry data of the respective robot segment, which can be utilized to further control the robot.

In addition to directly controlling the robot through contact, the sensor system's local resolution makes it possible to allocate special functions to particular regions, e.g. pushbuttons or sliders, which can be used to set the speed of movements or the robot's axial movement.

The sensor system's safety functions are not deactivated in the "haptic device mode". Should the active force exceed a threshold value, a switching signal is generated to stop robot movement. 


\section{Conclusion and Future Work}

Direct human-robot interaction and potential physical contact call for novel concepts and systems for safe robot movement. Broadening a robot's senses by enveloping it with an artificial pressure-sensitive skin is an effective measure to ensure robot systems are safe in such scenarios. Future work at the Fraunhofer IFF will expand this approach to robot safety. The artificial pressure-sensitive skin will play the main role.

The range of applications for this sensor system is clearly not limited to robotic applications. Further fields of application include machinery and plant safety and even the field of ambient assisted living where the sensor system can be employed in comfort tests to detect pressure [11].

\section{Literature}

[1] E. Schulenburg, N. Elkmann, M. Fritzsche, A. Girstl, S. Stiene and C. Teutsch. LiSA: A Robot Assistant for Life Sciences. In Proc. of the 30th Annual German Conference on Al (KI 2007), Osnabrück, Germany, September 2007

[2] M. Fritzsche, E. Schulenburg, N. Elkmann, A. Girstl, S. Stiene and C. Teutsch. Safe Human-Robot Interaction in a Life Science Environment. In Proc. of the IEEE International Workshop on Safety Security and Rescue Robotics (SSRR 2007), Rome, Italy, September 2007

[3] E. Schulenburg, N. Elkmann, M. Fritzsche and C. Teutsch. A Mobile Service Robot for Life Science Laboratories. 20. Fachgespräch Autonome Mobile Systeme (AMS 2007), Kaiserslautern, Oktober 2007

[4] S. Stiene and J. Hertzberg. Sicheres Navigieren in dynamischen Umgebungen mit 3DKollisionsvermeidung. In: K. Berns, T. Luksch (eds.): Autonome Mobile Systeme 2007. 20. Fachgespräch, Kaiserslautern, 18./19. Oktober 2007. Berlin (Springer) 2007, p. 68-74

[5] N. Elkmann, M. Fritzsche, E. Schulenburg, and C. Teutsch. LiSA: Ein Assistenzroboter für den Einsatz in Laborumgebungen zur sicheren Mensch-Roboter-Interaktion. In Proc. 5. VDI-Fachtagung ROBOTIK 2008 (June 11-12, Munich). vol. 2012 of VDI-Berichte, pages 227-230. VDI Wissensforum $\mathrm{GmbH}$, VDI Verlag GmbH Düsseldorf, 2008.

[6] MRK Systeme GmbH. Product description. http://www.mrksysteme.de/downloads/Prospekt_KR3_SI.pdf. Accessed December 2008

[7] De Luca, Alessandro; Albu-Schäffer, Alin; Haddadin, Sami; Hirzinger, Gerhard (2006): Collision Detection and Safe Reaction with the DLR-III Lightweight Manipulator Arm. In: Proceedings IROS 2006, S. 1623 - 1630, IEEE/RSJ International Conference on Intelligent Robots and Systems, Beijing, China, 2006-10-09 - 2006-10-15

[8] Woesch, T. Taktile Mensch-Maschine-Interaktion. In Robotik 2002 (=VDI-Berichte 1679), pp. 275280.

[9] Woesch, T. and Feiten, W. Reactive Motion Control for Human-Robot Tactile Interaction. In Proc. of the 2002 IEEE International Conference on Robotics and Automation (ICRA 2002), Washington D.C., USA, Mai 11-15, 2002, pp. 3807-3812.

[10]Weiss Robotics. $\quad$ Product http://www.weissrobotics.de/downloads/taktiles_sensorsystem.pdf. Accessed December 2008

[11]M. Fritzsche, M. Woitag, U. Amrhein, D. Berndt, N. Elkmann, R. Warnemünde, Neue textile Sensoren zur flächigen Druckerfassung, In Proc. of Ambient Assisted Living, 1. Deutscher Kongress mit Ausstellung (AAL 2008), Berlin, Germany, Februar 2008 